\title{
EL SOCIAL MEDIA MARKETING Y SU IMPACTO EN LA COMUNICACIÓN POLÍTICA EN CAMPAÑAS ELECTORALES DE ECUADOR
}

\author{
Ing. Rafer Cruz Mera PhD (c). \\ Universidad Laica Eloy Alfaro de Manabí \\ rafer.cruz@uleam.edu.ec \\ Ing. Temistocles Deodato Loor Chávez \\ PhD. Universidad Laica Eloy Alfaro de Manabí \\ deodato.loor@uleam.edu.ec \\ Ing. Rossana Sabando Mendoza Mg. \\ Universidad Laica Eloy Alfaro de \\ Manabí \\ estela.sabando@uleam.edu.ec
}

Ing. Ulises Mero Chávez Mg. Universidad Laica Eloy Alfaro de Manabí Para citar este artículo puede utilizar el siguiente formato:

Rafer Cruz Mera, Temistocles Deodato Loor Chávez, Rossana Sabando Mendoza y Ulises Mero Chávez: "El social media marketing y su impacto en la comunicación política en campañas electorales de Ecuador", Revista Caribeña de Ciencias Sociales (vol 10, № 7 julio-septiembre 2021, pp. 224-238. En línea:

https://doi.org/10.51896/caribe/EQDL8721

\section{RESUMEN}

Las nuevas tecnologías de la información y la comunicación han impactado al mundo moderno, convirtiéndose velozmente en una de las herramientas más poderosas y utilizadas por cualquier persona, en las actividades de conexión y comunicación. Actualmente el internet y las redes sociales son considerados los medios de comunicación más utilizado y de mayor eficacia a la hora de comunicarse e interrelacionarse con los demás. El objetivo de la investigación se centró en determinar de qué manera el social media marketing impacta en la comunicación política en campañas electorales de Ecuador. El enfoque utilizado es cualicuantitativo, permitiendo especificar características o descripciones de los medios de comunicación política que logran captar la atención a los electores, asimismo se describe claramente lo que buscan los electores y los medios de comunicación que influyen significativamente en la decisión del voto y el apoyo a un partido político. Mediante el programa SPSS 25, con el coeficiente de correlación p (Rho) de Spearman, se pudo comprobar la hipótesis general y específicas, demostrando que social media marketing impacta en la comunicación política en campañas electorales de Ecuador, además que las herramientas web, la promoción web y los C'S del social media impactan en la comunicación política en campañas electorales de Ecuador, alcanzando respectivamente los siguientes coeficientes de $(0,840),(0.763)$, $(0,880)$, y $(0,796)$, significando que se validan positiva y significativamente las hipótesis. 
Palabras clave: Social media marketing, redes sociales, comunicación política, electores, estrategias de comunicación.

\section{SOCIAL MEDIA MARKETING AND ITS IMPACT ON POLITICAL COMMUNICATION IN ELECTORAL CAMPAIGNS IN ECUADOR}

\section{ABSTRACT}

The new information and communication technologies have impacted the modern world, rapidly becoming one of the most powerful tools used by anyone in connection and communication activities. Currently the internet and social networks are considered the most used and most effective means of communication when communicating and interacting with others. The objective of the research focused on determining how social media marketing impacts political communication in electoral campaigns in Ecuador. The approach used is qualitative-quantitative, allowing to specify characteristics or descriptions of the political communication media that manage to capture the attention of voters, as well as clearly describing what voters and the media are looking for that significantly influence the decision to vote and vote. support for a political party. Using the SPSS 25 program, with Spearman's $p$ (Rho) correlation coefficient, the general and specific hypotheses could be verified, demonstrating that social media marketing impacts political communication in electoral campaigns in Ecuador, in addition to web tools, Web promotion and social media C's impact on political communication in electoral campaigns in Ecuador, reaching respectively the following coefficients of $(0.840),(0.763),(0.880)$, and $(0.796)$, meaning that they are positively and significantly hypotheses.

Keywords: Social media marketing, social networks, political communication, voters, communication strategies.

\section{INTRODUCCIÓN}

El presente artículo presenta algunas variables sobre la percepción política y su impacto en el marketing político. Sosteniendo que el predominio de la percepción política sobre la democracia se basa en el ámbito de la comunicación y las redes sociales, causando un impacto colosal en la sociedad política, los candidatos, los procesos de campañas electorales, así como también de las acciones positivas y negativas que contribuyen en la percepción y persuasión del target político ecuatoriano.

Actualmente, la comunicación política se enfoca en la aplicando de técnicas de marketing que permitan promover una determina imagen o un mensaje que se espera los potenciales electores perciban con agrado y genere el interés de participar en un determinado movimiento o partido político. Para Díaz Jiménez (2015), en Latinoamérica los partidos políticos por lo general no generan una pronta adaptación del marketing, lo que les permitiría incentivar una participación protagónica en la construcción de la sociedad por parte de los ciudadanos, lo que implica que se trabaje sobre la 
base de una ciudadanía, que con ayuda de las acciones de mercadotecnia promuevan una participación equilibrada por parte de las personas.

Gracias a la evolución de las herramientas tecnológicas, la comunicación 2.0 ha traspasado las fronteras de la web, permitiendo que la vida cotidiana se piense en términos de redes sociales como escenario de interactividad grupal e individual, como manifestación unipersonal y colectiva, y como forma de organización pública y privada. Siendo así que las redes sociales marcan un nuevo ambiente y arbotante comunicativo con un modelo de comunicación multidireccional.

Las redes virtuales en la comunicación política como una herramienta de éxito tuvieron su punto de partida en la campaña electoral del expresidente de Estados Unidos Barack Obama en el año 2008. Desde entonces año a año los políticos en sus campañas electorales para tener la posibilidad de ser escuchados han debido adecuarse a las nuevas tecnologías, a los cambios producidos en la sociedad y a las nuevas formas de comunicación políticos, usando cada vez más las redes sociales como estrategia fundamental de comunicación.

El marketing político nace como fruto de la relación entre la escuela de la mercadotecnia y la política. De acuerdo con (Martínez, 2014), se refiere al conjunto de técnicas de investigación, planificación, gerenciamiento y comunicación que se utilizan en el diseño y ejecución de acciones estratégicas y tácticas a lo largo de una campaña política, sea esta electoral o de difusión institucional. Por otro lado, Kotler y Kotler (1999) sustenta que una campaña electoral regida por normas del marketing político debe estar organizada de acuerdo con un modelo estructurado en la evaluación del ambiente electoral y el marketing estratégico, con el propósito de desarrollar los productos de comunicación de esta. En sí, el marketing político, se refiere al conjunto de actividades efectuadas a lo largo de una campaña para alcanzar un fin en particular, en donde la principal característica es la incorporación de acciones engendradas de la investigación de mercado, que determinen el modelo de producción de comunicación, así como las plataformas políticas y otras variables del proceso electoral.

Hoy en día no se puede entender la sociedad, sus modos de vida y sus problemas sin tener un ojo puesto en las redes sociales, es por ello por lo que estas nuevas herramientas de comunicación se han convertido en un elemento fundamental para mantener y mejorar las democracias actuales. Con la aparición de las redes sociales, la comunicación política se ha reescrito y ha evolucionado, consiguiendo que los medios, que años atrás eran los más eficaces, hoy hayan perdido capacidad de comunicación. La comunicación está estrechamente ligada con la acción política, de tal manera que el poder legislativo se abra a la sociedad y se comuniquen, expliquen y entiendan mejor todas las acciones a desarrollar, siendo esta la mejor forma de llegar a la sociedad y de ejercer la figura de representantes de los ciudadanos. Así también es la mejor manera para que los ciudadanos puedan fiscalizar el trabajo realizado.

Las personas procesan su experiencia social y política en la sociedad a partir de la acción comunicativa. Así encontramos a la esfera pública de la comunicación política, que anteriormente estaba localizada en clubs, salones, periódicos, libros y panfletos. Con la aparición de las nuevas 
tecnologías de comunicación y los ciberespacios, el concepto de esta esfera se redefine, señalándola como el espacio institucional donde se forma la voluntad política a través del flujo continuo de ideas, información, contestación, organización, discusión y lucha política, y en la que los ciudadanos se empoderan, pudiendo encontrar e intercambiar puntos de vista. Ante lo mencionado, se establece el siguiente objetivo: Determinar de qué manera el social media marketing impacta en la comunicación política en campañas electorales de Ecuador. Pudiendo examinar los efectos del marketing político, así como sus teorías como parte de la campaña política y de qué manera las redes sociales que actualmente se identifican como las mejores plataformas son usadas como nuevos y populares canales de comunicación sobre los votantes en las elecciones de diferentes dignidades políticas en Ecuador para conseguir el objetivo deseado.

\section{MARCO TEÓRICO}

\section{Social media marketing}

El social media marketing es el proceso mediante el cual las personas a través de los canales sociales online pueden promocionar sus sitios web, así como sus productos o servicios, permitiéndoles comunicarse con una colectividad mucho más grande a la que quizás no se tenía accesibilidad mediante los canales de publicidad tradicionales. (Weinberg, 2009, págs. 3-4).

El Marketing de Redes Sociales, es parte del marketing electrónico y se considera una herramienta, con la que se pretende mediante la utilización de redes sociales como MySpace, Facebook, Youtube, páginas web, entre otros, lograr la comunicación efectiva de una marca, servicio o producto. (San Millán Fernández, Medrano García, \& Blanco Jiménez, 2008, pág. 358).

El social media es el nombre que se le ha otorgado una de las redes sociales en relación al mercado en cuanto a promocionar y comercializar diversos productos o servicios, además estas redes son canales influyentes que ayudan a tener una mayor aproximación con el cliente. (Keinänen, 2015, pág. 2).

Podría decirse que este tipo de marketing se caracteriza por no ser invasivo, es decir, que pretende lograr sus objetivos a través de la influencia en espacios de intervención social en donde generalmente los contenidos son útiles e interesantes, pudiendo además mediante el mismo saber o tener una concepción más clara de las personas manifiestan en cuanto a una marca.

\section{Objetivos del social media marketing}

1. Notoriedad e Imagen de marca o "E-Reputación": gracias a la posibilidad de generar interacción en tiempo real, los medios sociales son excelentes vectores de información relevante a la marca.

2. Desarrollo comercial: una vez logrado establecer una imagen y notoriedad apropiada de una marca con valores asociados, así como un perfil de actuación empresarial concreto y unos interlocutores bien determinados y reconocidos, se crea una comunidad, misma que surge de la reacción positiva a los mensajes que emite la marca, convirtiéndose en de influencia positiva de esta. 
3. Compromiso de los miembros de la comunidad: en este punto se puede decir que se ha logrado llegar al grado superior de la estrategia de Social Media Marketing, ya que se ha conseguido transformar a los internautas en embajadores que protegerán la marca de cualquier ataque que hagan los detractores. (Bértol Gorospe, 2013)

\section{Tipos de medios sociales}

De acuerdo con Kaplan y Haenlein (2010), hay seis clases de medios sociales, mismos que se detallan a continuación:

1. Blogs. Sitios web creados para que cualquier individuo o empresa pueda publicar contenido en Internet de una manera sencilla, además permite que los lectores inserten sus comentarios sobre lo publicado. Los blogs pueden ser profesionales, personales, corporativos o de un nicho específico.

2. Microblogs. Se lo conoce también como nanoblogeo, en este caso este tipo de blogs permite a sus usuarios enviar y publicar mensajes muy cortos y concisos, que generalmente son solo de texto, como, por ejemplo: Twitter.

3. Redes sociales. Son estructuras formadas en internet que permiten que un grupo de personas con intereses comunes se comuniquen y compartan experiencias $u$ opiniones de manera ágil, y material digital, por ejemplo: Facebook.

4. Contenido compartido. En este caso este tipo de medio social, permite que los usuarios puedan publicar y compartir contenidos entre varios, por ejemplo: Youtube.

5. Contenido colaborativo. Permiten crear simultáneamente contenidos entre varios usuarios, por ejemplo: Wikipedia.

6. Realidad virtual. En este caso se crean mundos virtuales tridimensionales, en donde el usuario puede crear un personaje virtual, por ejemplo: Second life.

\section{Importancia del lenguaje persuasivo en la comunicación política}

De acuerdo con Boulton, (1978), la comunicación es un fenómeno complejo que continúa siendo un soporte vital de las relaciones y de la existencia humana, misma que se puede representar un arma de doble filo, al utilizarla ya sea de forma positiva o negativa (p. 41).

Por otro lado, Hallahan, Holtzhausen, Van Ruler, Vercic y Sriramish, (2007), manifiestan que la comunicación estratégica es el uso premeditado e intencionado de un mensaje que exterioriza una organización para cumplir una misión, que requiere de coherencia, coordinación de mensajes y tácticas entre los departamentos de una empresa.

La capacidad que tiene el hombre de usar el lenguaje para interactuar con su medio ambiente es una de las únicas características que lo diferencia de los animales. Por ello, este no solo debe verse como una fuente de cultura o un medio de expresión de pensamientos y sentimientos, sino que además debe considerarse como un sistema en el que cada elemento de la comunicación conduce su valor a partir del contexto total en el que funciona. Por tanto, se puede aseverar que nadie puede vivir sin comunicarse con otra persona, es por ello que el concepto del lenguaje es un 
recurso crítico en todas las actividades del ser humano, más aún en campañas políticas. Según Martín Barbero (1993, p. 27) el lenguaje influye en nuestro comportamiento, lo que hace que la cooperación humana sea posible, tomándolo como un medio a través del cual los las personas expresan sus sentimientos y deseos.

\section{Rol del internet en marketing político}

El marketing político es relativamente nuevo, pero ha crecido velozmente en los últimos años. Atrayendo hoy en día a muchos investigadores del campo de la comercialización y a los líderes de partidos políticos que empezaron a utilizar los instrumentos de marketing en sus campañas electorales, sobre todo a nivel de medios sociales, con el fin de persuadir a los votantes y centrarse en la influencia de los individuos respecto a los candidatos políticos para alcanzar el número deseado de votos (Kotler y Kotler, 1999).

Desde que apareció el internet a inicios de los 90, la población a nivel mundial en redes ha crecido descomunalmente, pasando de miles de visitas al mes a millones de visitas en días, o tan solo en horas. Es así que a medida que el panorama de las comunicaciones se torna más complejo y participativo, la población en red es cada vez más importante y tiene mayor poder, pudiendo acceder a la información de manera más fácil y teniendo la posibilidad de volverse en un participante activo del discurso público.

Para Newman, (1999), el marketing político es fundamental dentro de la política, sobre todo para las elecciones, referendos, gestión de servicios públicos, etc., ya que a través de las estrategias de mercadotecnia y comunicacionales utilizadas se puede alcanzar el triunfo de no solo a nivel comercial, sino además en las campañas políticas modernas.

\section{Nuevas formas de comunicación política}

Los nuevos medios de comunicación masiva son usados para hallar información y se convierten además en plataformas que permiten tener un foro de discusión. En el proceso político están impactando como un suministro de información y como una herramienta de democratización, ayudando así al votante a tomar decisiones más precisas. Es así que estos modernos medios se caracterizan por ser una fuente veloz de crecimiento de la información sobre las campañas, elecciones y los candidatos, superando notoriamente a la radio, los periódicos, aunque la televisión aún sigue siendo considerada una fuente principal de noticias políticas.

En los últimos años, la comunicación política ha tenido que cambiar drásticamente, debido a la influencia de extendido alcance en la política democrática. Actualmente, los políticos han enfocado sus esfuerzos, recursos y esnobismo en la comunicación con los ciudadanos. No obstante, los medios de comunicación tradicionales, como la radio y la prensa, siguen siendo las principales fuentes de información, pero que cada vez más se enfrentan a la reducción de la audiencia, que hoy en día tienen gustos e intereses muy volátiles. 
A nivel mundial, el uso de las redes sociales en las campañas electorales se ha intensificado significativamente, principalmente entre los adultos jóvenes. Para este grupo de edad es muy importante el aumento del uso de los medios sociales, ya que a través de estos se puede obtener información política, crear contenido generado por el usuario y expresar puntos de vista políticos. Además de poder evaluar cuáles son los efectos que tienen sobre el comportamiento y la participación política en las redes gracias al creciente uso de las redes de comunicación en el ámbito político.

El Internet funciona como eje central en la difusión con diversas inversiones privadas y por tanto distanciada de los otros medios de masa que generalmente han sido manipulados por los gobiernos de turno y los grandes grupos económicos, trayendo consigo una nueva manera de intercambio, haciendo que surja un ambiente forjado en la concepción de potenciar el desarrollo de un conglomerado de nuevas y creativas ideas con total libertad de expresión.

El internet a nivel social, cultural, artístico, económico y político ha causado una transformación impactante, por lo que, desde hace algún tiempo, tanto la política como la sociedad, están remodelándose permanentemente en un mundo dinámico e impredecible caracterizado por la tecnología. Gracias a su rápida expansión y capacidad de comunicar, el internet se utiliza como instrumento eficaz para la comunicación política, principalmente como instrumento electoral y como herramienta para crear líderes políticos. (Castro, 2008).

\section{Marketing político}

Para Saiz (2003) el marketing político o marketing electoral se refiere al conjunto de técnicas que sirve para identificar y conocer los deseos y necesidades del mercado electoral, además constituye un esquema ideológico que permite conocer el ofrecimiento de un candidato que apoyado por una campaña política personaliza dicho programa.

De acuerdo con López y Oregón, (2017), el marketing político, es la forma en la que se da a conocer un partido político que está en campaña electoral, en la que sus propuestas representen las perspectivas de los electores; para así lograr la aceptación, confianza y decisión del votante a favor del partido político que más se ajuste a la confiabilidad de llegar a satisfacer las expectativas del electorado.

Según Niffenegger (1989) el marketing político la forma en la que los partidos o candidatos, analizan las opiniones del entorno para generar e impulsar una oferta competitiva que ayude a lograr los objetivos que debe seguir la organización para alcanzar la satisfacción de los electores y conseguir votos (p.6)

El marketing político es una disciplina enfocada en estrategias, sobre cuándo y por qué se empieza una precampaña o campaña política, ya que una campaña electoral, debe seguir un orden secuencial que perita hacer las cosas bien y en el momento preciso. (Buró Creativo, 2010)

\section{Las redes sociales en la cibercampaña}


El internet como medio digital ha tenido un impacto significativo en la organización del marketing político, ya que además de hacer uso de la red para llegar a los nuevos votantes, ayuda a la extracción de datos y la manipulación de la opinión pública. La ciberpolítica es un término utilizado a nivel mundial, refiriéndose a la amplitud y alcance que tiene el Internet dentro de la actividad política. Es decir que se trata de un dominio de investigación en cuanto al rol de las nuevas tecnologías de la información en la vida política moderna y como son usadas por sus actores, así como los votantes utilizan los medios digitales para conocer más sobre los asuntos públicos.

El internet creó y a las TICs hoy en día existe la posibilidad de construir una comunicación política más cercana y más personalizada con los electores, pudiendo llegar con el mensaje a millones de votantes. Actualmente, en América Latina las cibercampañas se han convertido en la nueva forma de hacer política y de los procesos de consolidación democrática. (López, Gutiérrez e Islas, 2000).

Gracias a las nuevas tecnologías ha sido posible movilizar a muchos electores de diferentes edades a las urnas, lo que ha facilitan, la logística y la movilización electoral, con el propósito de triunfar en las elecciones. Por tanto, las cibercampañas no solo estudian el comportamiento de los votantes, sino que, también ha permitido mejorar la comunicación y la organización del partido. Además, las TICs se utilizan para transmitir los planes de gobierno y la imagen personal de los candidatos (Dader, Campos y Quintana, 2013).

\section{Hipótesis General}

- El social media marketing impacta en la comunicación política en campañas electorales de Ecuador.

\section{Especificas}

- Las herramientas web impacta en la comunicación política en campañas electorales de Ecuador.

- La promoción web impacta en la comunicación política en campañas electorales de Ecuador.

- Las c's del social media impacta en la comunicación política en campañas electorales de Ecuador.

\section{METODOLOGÍA}

El estudio tiene un enfoque cuali-cuantitativo, que de acuerdo a Hernández, Fernández y Baptista (2014), es cuantitativo "porque utiliza la recolección de datos para probar hipótesis con base a la medición numérica y el análisis estadístico" (p. 4). Asimismo, menciona que la investigación cualitativa se basa en "comprender los fenómenos, examinándolos desde la perspectiva de los participantes en un ambiente natural, en relación con su contexto", es decir que va de lo general a lo particular, describiendo las causas que generan la aparición del problema para luego proporcionar perspectivas teóricas, enfocándose en la lógica y luego en los procesos inductivos. (p. 358).

\section{Diseño de investigación}


Se utilizó un instrumento de medición en escala de Likert, de tipo no experimental. Para Hernández, Fernández y Baptista, (2014), manifiestan que, para conseguir datos relevantes y sustanciales del objeto de estudio, la información se acoge en un determinado momento. (p.40).

\section{Unidad de análisis}

Se toma como unidad de análisis a la población que habita en Ecuador, misma que de acuerdo con el último informe del Instituto Nacional de Estadísticas y Censos (2020), es de 17’510.643.

\section{Población de estudio}

De acuerdo a Pérez (2005), por varios factores, el muestreo de toda población objetivo de estudio no siempre es posible, puesto que en ocasiones estos no permiten obtener información de algunos de sus elementos ya sea por usencia, inaccesibilidad o negativa a colaborar.

De acuerdo a Arias y Peñaloza (2013), estadísticamente el sometimiento de la población finita o infinita a la investigación, dependerá de la posibilidad que tenga el investigador de contar con un listado completo de los sujetos a ser estudiados. Para la presente investigación, la población está compuesta por 17'510.643 habitantes en Ecuador.

Tabla 1:

Población de Ecuador

\begin{tabular}{|r|r|r|}
\hline Hombres & Mujeres & Población Total \\
\hline 8.665 .937 & 8.844 .706 & $17^{\prime} 510.643$ \\
\hline
\end{tabular}

Fuente: Instituto nacional de estadística y censos (INEC). Proyección poblacional (2020) - Adaptado por autores.

\section{Dimensión de la muestra}

De acuerdo con las características del estudio fue necesario aplicar el muestreo probabilístico aleatorio simple, considerando el margen de error y el nivel de confianza. Se aplicó la siguiente fórmula para calcular la muestra:

$\mathbf{n}=\frac{Z^{2} \cdot P \cdot \mathbf{Q} \cdot \mathbf{N}}{Z^{2} \cdot P \cdot \mathbf{Q}+(\mathbf{N})(\mathrm{e})^{2}}$

Dónde:

$\mathrm{N}=$ Población o universo $(6.430 .029)$

$\mathrm{n}=$ Tamaño de la muestra (?)

$Z=$ Nivel de confianza (1.96)

$\mathrm{P}=$ Probabilidad de aceptación (0.5)

$\mathrm{Q}=$ Probabilidad de rechazo $(0.5)$

$\mathrm{e}=$ Margen de error de muestreo (0.05)

$\mathrm{n}=\frac{1.96^{2} *(0.5) *(0.5) *\left(17^{*} 510.643\right)}{1.96^{2} *(0.5) *(0.5)+\left(17^{*} 510.643\right) *(0.05)^{2}}$

$\mathrm{n}=\frac{3.8416 * 0.25 * 17^{*} 510.643}{3.8416 * 0.25+17^{*} 510.643 * 0.0025}$ 


$$
\mathrm{n}=\frac{16,817,221.5372}{42,043.0538}=400
$$

De acuerdo con los resultados después de aplicar la formula, el tamaño de la muestra es de 400 personas a encuestar.

\section{Recolección de datos}

Por medio de una encuesta compuesta por 40 preguntas, estructurada en escala de Likert y aplicada a 400 personas, se lograron recolectar los datos necesarios para cumplir con el objetivo del estudio. El instrumento fue validado por expertos, en el que se analizó la validez de contenido, criterio y constructo, así como también por medio de programa estadístico SPSS.

\section{RESULTADOS}

Mediante la aplicación del programa estadístico SPSS, se procedió al uso del coeficiente Alfa de Cronbach para establecer la fiabilidad del instrumento, con el cual de acuerdo al de acuerdo al baremo estadístico clásico, se demuestra una alta confiabilidad, con un valor de 0,847.

\section{Tabla 2.}

Coeficiente de fiabilidad Alfa de Cronbach

\begin{tabular}{rr}
\hline \multicolumn{2}{c}{ Estadísticas de fiabilidad } \\
\hline Alfa de Cronbach $\mathrm{N}$ de elementos \\
, 847 & 40 \\
\hline
\end{tabular}

Recurso: Programa estadístico SPSS - 25

\section{Uso del Rho de Spearman para para medir correlación}

Para comprobar las hipótesis, se recurrió al análisis de coeficiente de correlación de Rho de Spearman, mediante el programa estadístico SPSS. Para el análisis e interpretación de los resultados es importante tener en cuenta el baremo de medición, descrito en la siguiente tabla:

\section{Tabla 3}

Grado de relación según coeficiente de correlación

\begin{tabular}{|c|c|}
\hline RANGO & RELACIÓN \\
\hline-0.91 a -1.00 & Correlación negativa perfecta \\
\hline-0.76 a -0.90 & Correlación negativa muy fuerte \\
\hline-0.51 a -0.75 & Correlación negativa considerable \\
\hline-0.11 a -0.50 & Correlación negativa media \\
\hline$-0.01 \mathrm{a}-0.10$ & Correlación negativa débil \\
\hline 0.00 & No existe correlación \\
\hline$+0.01 \mathrm{a}+0.10$ & Correlación positiva débil \\
\hline$+0.11 \mathrm{a}+0.50$ & Correlación positiva media \\
\hline$+0.51 \mathrm{a}+0.75$ & Correlación positiva considerable \\
\hline$+0.76 \mathrm{a}+0.90$ & Correlación positiva muy fuerte \\
\hline$+0.91 \mathrm{a}+1.00$ & Correlación positiva perfecta \\
\hline
\end{tabular}

Fuente: Elaboración autores, basada en Hernández Sampieri \& Fernández Collado, 1998

\section{Comprobación de hipótesis}


Tabla 4.

Resultados prueba de hipótesis

\begin{tabular}{|c|c|c|c|c|c|}
\hline Hipótesis & $\begin{array}{c}\text { Variable } \\
\text { Independiente }\end{array}$ & $\begin{array}{c}\text { Variable } \\
\text { Dependiente }\end{array}$ & Sig & $\begin{array}{c}\text { Rho de } \\
\text { Spearman }\end{array}$ & Resultado \\
\hline H. General & $\begin{array}{l}\text { X. Social media } \\
\text { marketing }\end{array}$ & & ,000 & ,840 & Se rechaza Ho \\
\hline H. Específica 1 & X1. Herramientas web & Y. & ,000 & ,763 & Se rechaza Ho \\
\hline H. Específica 2 & X2. Promoción web & $\begin{array}{c}\text { Comunicación } \\
\text { política }\end{array}$ & ,000 & ,880 & Se rechaza Ho \\
\hline H. Específica 3 & $\begin{array}{l}\text { X3. Las C's del social } \\
\text { media }\end{array}$ & & ,000 & ,796 & Se rechaza Ho \\
\hline
\end{tabular}

Recurso: Programa estadístico SPSS - 25

Demostraciones e interpretaciones de acuerdo con la tabla \# 4

H. General (X)

Hipótesis General

Ho: El social media marketing NO impacta en la comunicación política en campañas electorales de Ecuador.

H1: El social media marketing SI impacta en la comunicación política en campañas electorales de Ecuador.

\section{Análisis de prueba}

La validación de la hipótesis general (X), procesados los datos, nos muestra una significación de 0,00 y un coeficiente p (Rho) Spearman de 0,840, afirmándose la hipótesis, lo cual evidencia un nivel de correlación "alta o significativa” en el cruce de variables.

\section{Hipótesis Específica 1 (X1)}

Ho: Las herramientas web NO impactan en la comunicación política en campañas electorales de Ecuador.

H1: Las herramientas web SI impactan en la comunicación política en campañas electorales de Ecuador.

\section{Análisis de prueba}

El procesamiento de datos en el SPSS 25 para la hipótesis específica (X1) establece una significación bilateral de 0,00 con un coeficiente de $\mathrm{p}$ (Rho) de Spearman de 0,763, ratificando positivamente el supuesto, tal como lo demuestra el nivel de correlación.

\section{Hipótesis Específica 2 (X2)}


Ho: La promoción web NO impacta en la comunicación política en campañas electorales de Ecuador. H1: La promoción web SI impacta en la comunicación política en campañas electorales de Ecuador.

\section{Análisis de prueba}

Los resultados de la hipótesis especifican (X2), exponen una significación bilateral de 0,00 con un coeficiente $p$ (Rho) de Spearman de 0,880, por lo que se afirma el supuesto.

\section{Hipótesis Específica 3 (X3)}

Ho: Las C'S del social media NO impactan en la comunicación política en campañas electorales de Ecuador.

H1: Las C'S del social media SI impactan en la comunicación política en campañas electorales de Ecuador.

\section{Análisis de prueba}

La hipótesis (X3), de acuerdo con el estadístico SPSS 25, demuestran una significación bilateral de 0,00 con un coeficiente $p$ (Rho) de Spearman de 0.796, por lo que se acepta la hipótesis, con una alta significancia.

\section{DISCUSIÓN}

Debido a que la actividad política actualmente se enfoca principalmente en comunicar, los actores políticos modernos se ven preocupadas por ser beligerantes en la comunicación, por lo que precisan que un mensaje sea correctamente emitido, neutralizado o derrotado. No obstante, es preciso entender que la comunicación política no siempre debe estar necesariamente dirigida a ganar una elección, ya que también puede utilizarse para una campaña política gubernamental, un referéndum, así como además para sacar una ley o a su vez para derogarla.

La lucha por alcanzar el poder entre los partidos políticos, los diversos factores inmersos en este sistema, y particularmente el nuevo entorno de los medios de comunicación, han sido los elementos determinantes para la aparición del marketing político, ya que los medios con sus reglas comerciales, no les han dejado otra alternativa que adaptarse a sus criterios, obligándolos por tanto a entrar en escena mediante el uso del marketing como una respuesta ante la capacidad de influir sobre los medios en el espacio de la comunicación política. En definitiva, el marketing político se puede apreciar como un resultado del poder de los medios para influenciar en la comunicación política y en la opinión pública. (Trejo, 1991).

En Ecuador, por raíces e idiosincrasias, los votantes generalmente no sufragan de forma racional, es decir que a la hora de decidir por quien votar, basan sus decisiones en emociones y sentimientos. Es por esto que hoy en día el marketing a nivel político juega un rol fundamental, marcando una nueva tendencia, estilo o manera de hacer política, donde tanto el partido como los grupos de interés cobran gran importancia, ante lo cual se vuelve muy importante que las actividades que desarrolle el candidato en este sentido sean permanentes. 
El marketing, ayuda a generar una comunicación abierta entre el candidato y el elector, en donde las tecnologías de la información y comunicación, a través de los medios masivos como las redes sociales, son actualmente el instrumento más valioso con el que puede contar un político. Por ello, es preciso considerar a las herramientas del social media marketing como elemento clave de la comunicación política es inevitable para emprender una campaña electoral, ya que esta se encarga de comunicar apropiadamente las propuestas de gobierno que plantean los movimientos políticos.

\section{CONCLUSIONES}

En la mercadotecnia política el mercado está conformado por la población, para quienes sus necesidades son la base para formular las estrategias que se deben seguir en una campaña electoral o política. Por ello, el desarrollo de una buena campaña comunicacional haciendo uso de las herramientas de marketing y de las TICs, puede marcar la diferencia entre proyectar una imagen positiva o negativa del postulante ante los votantes.

A través del programa estadístico SPSS, se pudo validar la hipótesis general, cuyos resultados con un $p$ (Rho) de Spearman de 0,840, evidencian la correlación entre las variables principales del estudio. Es decir que el social media marketing impacta en la comunicación política en campañas electorales de Ecuador, por ello los candidatos o partidos políticos de deben aplicar estrategias de marketing y hacer uso de las herramientas web como factor clave de comunicación, para sí poder alcanzar los objetivos planteados.

Además, se pudo validar la hipótesis específica 1, con un $p$ (Rho) de Spearman de 0,763, demostrando que las herramientas web impactan en la comunicación política en campañas electorales de Ecuador, es decir que son instrumentos valiosos a la hora de comunicar la propuesta política de cada candidato.

De la misma manera, en la hipótesis específica 2, el p (Rho) de Spearman resultó con un valor positivo significativo de 0,880 , validando la misma, en donde se determinó que la promoción web impacta en la comunicación política en campañas electorales de Ecuador, por lo que las campañas políticas deben manejar mensajes efectivos que cumplan con las expectativas de los electores para así poder sumar adeptos al partido.

Concluyentemente, en la hipótesis especifica 3, se valida el supuesto, con un $\mathrm{p}$ (Rho) de Spearman positivo significativo de 0,796 , demostrando que la imagen de marca influye en las emociones de los consumidores ecuatorianos, por lo que es preciso que las c's del social media impacta en la comunicación política en campañas electorales de Ecuador.

\section{REFERENCIAS}

Arias, S. y Peñaloza, M. (2013). Muestreo. Enfoque ilustrado para investigar. Grupo de investigación en Evaluación y Mercadeo EVMERGI. Universidad de Los Andes. Venezuela.

Bértol Gorospe, H. Z. (2013). Guía del Social Media Marketing: ¿Cómo hacer gestión empresarial 2.0 a través de la aplicación de Inteligencia Digital? Madrid, España: ESIC 
Boulton, M. (1978). La anatomía del lenguaje: Diciendo lo que queremos decir. Londres: Routledge y Kegan Paul.

Buró Creativo, S. d. (2010). Marketing Politico. Mexico.

Cotarelo, R. y Olmeda, J. A. (2014). La democracia del siglo XXI. Política, medios de comunicación, internet y redes sociales, p. 501.

Dader, J. L.; Campos, E. y Quintana, N. (2013). Las webs de los partidos en la campaña de 2011: Una panorámica cualitativa de su actividad y un análisis cuantitativo de su transparencia. Vid. Crespo, I. (2011). Partidos, medios y electores en procesos de cambio.

Díaz Jiménez, O. (2015). Marketing político y profesionalización de las campañas electorales presidenciales del Partido Acción Nacional y del Partido de la Revolución Democrática, 19942006.

Hallahan, K.; Holtzhausen, D.; Van Ruler, B.; Vercic, D. y Sriramesh, K. (2007). Defining strategic communication. International Journal of Strategic Communication, 1 (1), pp. 3- 35.

Hernández Sampieri, R., \& Fernández Collado, C. (1998). Metodología de la investigación. México: McGraw-Hill.

Hernández, R., Fernández, C. y Batista, P. (2014). Metodología de la Investigación. Ed. MacGrawHill.México.

Instituto Nacional de Estadísticas y Censos. (2020). Informe poblacional anual. INEC. Ecuador.

Kaplan, Andreas M. Haenlein, Michael. (2010). (Pg. 61). Los usuarios de los países, junidos! Los retos y oportunidades de las redes sociales. Business Horizons. Paris, Francia. (En línea). Fecha de consulta (12-07-2013).

Keinänen, H. \&. (2015). Antecedents of social media B2B use in industrial marketing context: Customers' view. . The Journal of Business \& Industrial Marketing, 711-722.

Kotler, P. and Kotler, N. (1999). Political Marketing, in B. I. Newman (Ed.), Handbook of Political Marketing, Sage, Thousand Oaks, pp. 3-18

López, C.; Gutiérrez, F. e Islas, O. (2000). Las cibercampañas independientes. Revista Latina de Comunicación Social, septiembre 33(3). Extraída el 30/VIII/2011 desde http://www.ull.es/ publicaciones/latina/aa2000kj//x33se/53islas/octavio.htm

López y Oregón (2017). Del marketing político a las comunidades de marca. Un estudio comparativo de partidos políticos en Bogotá D.C. Revista Universidad y Empresas. Bogotá - Colombia.

Martín Barbero, Jesús (1993), De los medios a las mediaciones. Comunicación, cultura y hegemonía, Barcelona: Gustavo Gili.

Martínez Pandiani, G. (2014). Political Marketing, Campaigns, Media and Electoral Strategies, Buenos Aires-Argentina: Editor U German S.A.

Newman, B. I. (1999). Handbook of Political Marketing, Sage, Thousand Oaks

Niffenegger, Phillip B. (1989): Strategies for Success from The Political Marketers. The Journal of Consumer Marketing; Winter 1989; 6, 1 pg. 45-51

Pérez, C. (2005). Muestreo Estadístico. Conceptos y problemas resueltos. Madrid: Pearson

Saiz, F. J. (2003). Marketing político. Rioja: Escuela Superior de Gestión Comercial y Marketing, ESIC. 
San Millán Fernández, E., Medrano García, M. L., \& Blanco Jiménez, F. J. (2008). Social media marketing, redes sociales y metaversos. Universidad de La Rioja, 358

Trejo, Raúl (1991) Campañas y elecciones en la prensa en la ciudad de México. En P. Arredondo, G. Fregoso y R. Trejo D. (eds.), Así se calló el sistema. Comunicación y elecciones en 1988, México: Universidad de Guadalajara

Weinberg, T. (2009). The New Community Rules: Marketing on the Social Web. New York, Estados Unidos: "O'Reilly Media, Inc. 